\section{e0359 A CARDIAC SODIUM CHANNEL SCN5A SUBUNIT GENE POLYMORPHISM AND EARLY REPOLARISATION VARIANT}

doi:10.1136/hrt.2010.208967.359

Ying Gao, Ping Zhang, Ying Tian, Jihong Guo. Beijing University People's Hospital

Objectives To investigate the effect of the PolymorPhism of cardiac sodium channel subunit $\alpha$ (SCN5A) gene on early repolarisation variant (ERV)

Methods Using PCR direct sequencing technology, two single nucleotide PolymorPhisms (SNP) of SCN5A gene, 1673 A>G and $3666+69$ G>C 84, was analysed by detecting genetic variation genotyPe and allele frequency distribution in 54 early repolarisation variant and 30 healthy subjects from the Beijing MuniciPal PeoPle's HosPital of Peking University and the Sixth HosPital of Beijing.

Results In the $1673 \mathrm{~A}>\mathrm{G}$ locus, there is no significant difference in genetic mutation as well as allele frequency distribution between variant and healthy grouP. In $3666+69 \mathrm{G}>\mathrm{C}$ locus, both of genetic variation genotyPe and allele frequency distribution in variant grouP are significantly different from control grouP $(\mathrm{p}<0.05)$. In variant grouP, there is no statistical difference in sex, syncoPe and the J-wave elevation range.

Conclusion $3666+69$ G gene PolymorPhism $(\mathrm{G} \rightarrow \mathrm{C})$ may be associated with early repolarisation variant.

\section{e0360 THE STUDY OF THE DIAGNOSTIC VALUE FOR ISCHAEMIA MODIFIED ALBUMIN (IMA)}

doi:10.1136/hrt.2010.208967.360

Liu Zhiqin1, 'Hang Shang, ${ }^{1}$ An Yapin, 'Li Jun, ${ }^{2}$ Liu Chang. ${ }^{1}$ People's Hospital of Guizhou Province; ${ }^{2}$ Medical Doctor, Medical college of Zhongshan University

Objective To study the diagnostic value of ischaemia modified albumin (IMA) for unstable angina (UA).

Methods The level of blood serum IMA of UA patient, stable angina (SA) patient and the non- coronary heart disease group (CHD) were detectioned by IMA reagent kit (Changsha YIKANG Technical Co. Ltd). To calculate the IMA diagnostic sensitivity, ecificity positive predictive value (PPV) and negative predictive value (NPV) of UA. IMA goes by albumin cobalt binding (ACB), IMA value was denoted by $A B C$ value. The lower $A B C$ value was, the higher free cobaltion $\left(\mathrm{Co}^{2+}\right)$ concentration was, to indicate higher IMA. ABC value was shown by $\mathrm{U} / \mathrm{ml}$, one $\mathrm{U}$ was defined as $1 \mu \mathrm{g} \mathrm{Co}{ }^{2+}$ absorbed by albumin in $1 \mathrm{ml}$ serum. The optimal cutoff point (cutoff)as distinguishing UA from The control group was obtain By receiver operator characteristive (ROC).

Results 1. ABC value of UA group (72 patients) was $62.80 \pm 9.56 \mathrm{U} /$ $\mathrm{ml}$, SA group (43 patiens) was $73.56 \pm 6.43$, non-CHD group (39 patients) was $76.13 \pm 7.25$. The $\mathrm{ABC}$ value in the UA group was obviously lower than the SA group and the non-CHD group $(p<0.01)$, but the SA group was not difference from the non-CHD group ( $p>0.05)$. The ABC value in the UA group within $6 \mathrm{~h}(43$ patients) were obviously lower than that in the period from 6 to $12 \mathrm{~h}$ (29 patients) ( $\mathrm{p}<0.01)$, the latter was also obviously lower than the non-CHD group $(p<0.01)$. That indicated: The level of IMA in the group of UA patients within $12 \mathrm{~h}$ was obviously higher than the SA group and the non-CHD group $(p<0.01)$, which was more significantly higher within $6 \mathrm{~h}$. 2. The area under cure (AUC) was 0.945 , cutoff point was $66.3 \mathrm{U} / \mathrm{ml}$, the sensitivity of the diagnosis of UA patient onset within $12 \mathrm{~h}$ is $91.67 \%$, the specifity is $82.05 \%$., PPV is $90.41 \%$, NPV was $84.21 \%$. That indicated : the sensitivity and PPV of IMA diagnosis of UA patient onset within $12 \mathrm{~h}$ were high, the specifity and NPV were lower.

Conclusion IMA becomes possibly the sensitive biochemical marker for myocardial ischaemia and is applyed diagnosing UA in the earlier period. e0361 EFFECT ON ELECTROLYTIC TO COMBINING APPLICATION OF CALCIUM CHANNEL BLOCKER (CCB) AND DIURETIC FOR GENERAL HYPERTENSIVES

doi:10.1136/hrt.2010.208967.361

Xie Xiaobing, Hong Jing, Tao Sha, Li Nanfang. Hypertension Unit of The People's Hospital of Xinjiang Uygur Autonomous Region, The Institute of Hypertension of Xinjiang, The Center of Hypertension of The People's Hospital of Xinjiang Uygur Autonomous Region

Introduction To evaluate the effects of low-dose applications of dihydrochlorothiazede (DHCT) or DHCT and nitrendipine on blood pressure, heart rate, and serum electrolytes in hypertensives in Xinjiang agriculture-pasture region.

Method Administer low dose DHCT two weeks of hypertensive disease in basic level region, according to blood pressure reach standard, combining nitrendipine (68 subjects), keep on low dose DHCT (67 subjects), follow-up visit for 3 months, to observe change of electrolytic and blood pressure (BP), heart rate (HR).

Result BP and HR decreased significantly after antihypertensive drug therapy compared with the baseline level each regimen $(p<0.05)$. According to the post-treatment by trimenon, Blood sodium of each regimen after antihypertensive drug therapy was lower than that before antihypertensive drug therapy, $\mathrm{p}<0.05$ There were no statistical differences in changes of electrolytic $\left(\mathrm{Na}^{+}\right.$, $\mathrm{K}^{+}$and $\mathrm{Ca}^{+}$)between two regimens, $\mathrm{p}>0.05$.

Conclusion They can lead to hyponatremia low dose regimen of DHCT and combiniant nitrendipine towards to hypertensive disease in basic level region. But on the basis of low dose regimen of DHCT combining nitrendipine is not to further increase the electrolytic turbulence.

\section{e0362 CORRELATION BETWEEN ATHEROSCLEROTIC PLAQUES MORPHOLOGY AND SEROUS HYPERSENSITIVE C-REACTIVE PROTEIN IN PATIENTS WITH PREMATURE CORONARY HEART DISEASE}

doi:10.1136/hrt.2010.208967.362

Lijian Gao, Jue Chen, Jilin Chen, Juejin Yang, Shubin Qiao, Bo Xu, Jianjun Li, Xuewen Oin, Min Yao, Yongjian Wu, Jinqing Yuan, Shijie You, Yu Chen, Jun Dai, Jie Qian, Runlin Gao. Fuwai Hospital

Objectives To investigate the relationship between intravascular ultrasound (IVUS) imaging characteristics and plasma hypersensitive C-reaction protein of premature coronary heart disease (CHD).

Methods Comparative study was conducted on 57 premature $\mathrm{CHD}$ and 57 late CHD patients whose data were intact and could be analysed, and the clinical follow-up was completed at 12 months after the procedure.

Results Comparison between premature CHD patients and late CHD patients, the ratio of lipid core measured by IVUS to plaque of coronary artery was in linear relationship with plasma hypersensitive CRP in premature CHD patients $(p<0.001)$. we found that the former has more patients with family history of $\mathrm{CHD}$, smoking, myocardial infarction, hyperlipaemia and unstable angina pectoris (all the $\mathrm{p}$ value $<0.05$ ), while the latter has more patients with diabetes. There was no difference between the two groups on the morphology of the lesions by coronary artery angiography. But lesion extent, cross-sectional areas surrounded by external elastic membrane, eccentricity index (EI), plaque plus medial area (MA), positive remodelling, plaque burden and the ratio of lipid core to plaque by IVUS were usually seen in the former group (all the $p$ value $<0.05$ ). There was no difference on major adverse cardiac events (MACE) rate between the two groups at 12-month clinical follow-up. The MACE rate was $7.89 \%$ (9/114). Three and four patients suffered from TVR or TLR and two patients died. There was only 1 patient died from late stent thrombosis in the two groups $(0.87 \%)$. 